\title{
Légitimer des technologies controversées : le rôle croissant des spécialistes et des scientifiques dans la communication d'entreprise en ligne
}

Legitimizing controversial technologies: the increasing role of specialists and scientists in online corporate communication

\section{Fanny Domenec}

\section{OpenEdition \\ Journals}

Édition électronique

URL : http://journals.openedition.org/asp/4951

DOI : 10.4000/asp.4951

ISSN : 2108-6354

\section{Éditeur}

Groupe d'étude et de recherche en anglais de spécialité

\section{Édition imprimée}

Date de publication : 1 mars 2017

Pagination : 93-121

ISSN : 1246-8185

Référence électronique

Fanny Domenec, « Légitimer des technologies controversées : le rôle croissant des spécialistes et des scientifiques dans la communication d'entreprise en ligne », ASp [En ligne], 71 | 2017, mis en ligne le 01 mars 2018, consulté le 01 novembre 2020. URL : http://journals.openedition.org/asp/4951 ; DOI : https://doi.org/10.4000/asp.4951

Ce document a été généré automatiquement le 1 novembre 2020.

Tous droits réservés 


\title{
Légitimer des technologies
} controversées : le rôle croissant des spécialistes et des scientifiques dans la communication d'entreprise en ligne

\author{
Legitimizing controversial technologies: the increasing role of specialists and \\ scientists in online corporate communication
}

\section{Fanny Domenec}

\section{Introduction}

1 Si l'on ne pense pas d'emblée aux scientifiques ou aux ingénieurs en tant qu'acteurs de la communication d'entreprise, ils jouent pourtant désormais un rôle prépondérant à ce niveau dans les multinationales spécialisées dans l'innovation technologique et scientifique. Cette étude porte sur la visibilité croissante des chercheurs, des médecins ou des ingénieurs au sein d'entreprises américaines spécialisées dans les secteurs de l'agrochimie, du pétrole, du nucléaire et de la pharmacie. Il s'agit de déterminer si cette catégorie de spécialistes constitue une communauté discursive propre à des secteurs marqués par la controverse, en réponse à des visées communicationnelles spécifiques. Dans cette étude, par commodité et économie, nous rassemblons ces acteurs particuliers sous l'étiquette "spécialistes", même si l'on imagine bien que d'autres acteurs de l'entreprise peuvent, eux aussi, revendiquer une certaine spécialisation dans leur propre secteur (ressources humaines, gestion, etc.).

2 Cette étude s'inscrit dans la lignée de travaux de recherche précédents (Domenec 2013, 2015) sur le milieu, le discours et la culture des multinationales américaines spécialisées dans des activités controversées: pétrole et gaz de schiste (Chevron, 
ExxonMobil), agrochimie et organismes génétiquement modifiés (Monsanto, Dow AgroSciences), nucléaire (Duke Energy, Entergy) et industrie pharmaceutique (Merck, Pfizer). Ces multinationales présentent la particularité de se trouver à l'intersection du monde de l'entreprise et de domaines scientifiques et/ou technologiques controversés. Il convient de s'interroger sur la manière dont cette double spécialisation influence le discours institutionnel, notamment à travers l'importance croissante des spécialistes dans la communication d'entreprise. Il semble en effet que ces derniers présentent un exemple particulièrement intéressant d'acteurs "plurispécialisés", dont la communication mêle les codes du discours institutionnel de l'entreprise et du discours scientifique et technique propre à une communauté de chercheurs ou d'ingénieurs. Afin de caractériser leur fonction au sein du discours de l'entreprise, deux corpus ont été réunis et analysés.

Le premier corpus d'étude comprend les pages d'accueil des entreprises étudiées, mises en ligne entre 2000 et $2015^{1}$. Les pages d'accueil les plus anciennes ont été retrouvées sur le site Wayback Machine, qui permet aux utilisateurs d'archiver les pages Internet de leur choix à la fréquence qu'ils souhaitent. Une analyse du contenu visuel et textuel a été effectuée, afin d'examiner l'évolution des références aux sciences et aux techniques et l'insertion progressive de l'image de l'expert sur les sites Internet des entreprises. Le second micro-corpus comprend des genres émergents récemment mis en place par les multinationales pour permettre aux spécialistes de présenter leur mission au sein de l'entreprise, à travers des entretiens, des vidéos, des pages-produits ou des blogs. Il s'agit de caractériser la forme et le contenu des supports numériques sur lesquels interviennent les spécialistes d'entreprise, afin de mieux comprendre leur rôle au niveau du discours de l'entreprise. L'analyse du contenu textuel de ces genres émergents porte sur la redéfinition de l'image de l'entreprise et de ses activités, à travers des procédés de reformulation ou de simplification.

Cet article est organisé comme suit : tout d'abord, les notions de risque technologique, de vulgarisation et de médiation sont présentées. L'approche chronologique permet ensuite de comprendre comment les scientifiques d'entreprise sont progressivement devenus des ambassadeurs indispensables du milieu spécialisé. Enfin, les résultats de l'analyse de corpus montrent que les spécialistes font désormais office de médiateurs de l'innovation technologique, dans le but de légitimer des activités controversées.

\section{Risque technologique, vulgarisation et médiation : définitions}

5 Il semble ici nécessaire de préciser ce que nous entendons par l'expression « risque technologique ». De nos jours en effet, la plupart des entreprises doivent faire face à différents types de risque, parmi lesquels les risques naturels, les risques systémiques, les risques opérationnels ou les risques industriels (Hassid 2011:16; Beitone et alii 2010 : 408). En finance d'entreprise, la gestion du risque (risk management) couvre, entre autres, les risques de crédit, de liquidité ou opérationnels. Enfin, dans le domaine de la gestion des entreprises, l'acronyme VUCA, pour volatility, uncertainty, complexity, ambiguity (Bennet \& Lemoine 2014), fait référence à la combinaison de différents facteurs de risque : volatilité, incertitude, complexité et ambiguïté. 
6 Dans le cadre de cette étude, nous nous interrogeons sur la nature particulière du «risque technologique », considéré comme propre aux entreprises spécialisées dans des secteurs impliquant des sciences ou des techniques controversées. Contrairement aux risques mentionnés précédemment, il semble que le risque technologique n'est pas circonscrit aux résultats financiers de l'entreprise mais implique également l'ensemble de la société, à travers les impacts potentiels des activités des entreprises du pétrole, de l'agrochimie, du nucléaire ou de la pharmacie sur la santé humaine ou sur l'environnement. Le risque technologique est ici considéré comme un élément de spécialisation contingent, lié à une pression environnementale exacerbée au cours des dernières années. Suivant la définition des domaines spécialisés comme «un ensemble de connaissances et/ou de pratiques qui transcendent leurs communautés d'origine et sont mises au service d'un objectif particulier ${ }^{2}$ » (Van der Yeught 2016:51), nous souhaitons poser l'existence d'un domaine spécialisé du risque technologique, qui serait transversal au milieu professionnel de l'entreprise caractérisé par des activités spécifiques « sujettes à des déterminations spatiales et temporelles ${ }^{3}$ » (ibid.).

7 Des recherches préliminaires conduites sur six entreprises américaines du pétrole et de l'agrochimie ont montré que le risque constitue un élément de spécialisation au sein du monde de l'entreprise (Domenec 2013), qui « définit la place reconnaissable de [ces entreprises] au sein de la société [...] et détermine [leur] composition et [leur] organisation spécifiques» (Petit 2010 : § 20). En effet, des analyses de différents genres $\mathrm{du}$ discours de l'entreprise et des entretiens avec des membres des multinationales étudiées ont permis de conclure qu'en réponse aux controverses technologiques et environnementales autour des énergies fossiles, des pesticides ou des OGM, Monsanto, Dow Agrosciences, DuPont Pioneer, ExxonMobil, Chevron et Conoco Phillips privilégient une stratégie de légitimation systématique de leurs activités. Au-delà de la promotion de biens et de services, il s'agit avant tout de conserver un droit à opérer (license to operate ou social license to operate, SLO, Owen \& Kemp 2013:30) aux yeux du public et de la société.

$8 \mathrm{Au}$ sein de ce processus de légitimation, les chercheurs, les médecins ou les ingénieurs occupent une place fondamentale, puisqu'ils combinent une spécialisation disciplinaire (en tant que spécialistes de leur domaine technique et/ou chercheurs) et une spécialisation professionnelle (en tant que membres de l'entreprise). La notion de spécialiste varie en fonction du domaine d'activité : il peut s'agir de médecins pour les entreprises pharmaceutiques, d'ingénieurs pour les entreprises du pétrole ou du nucléaire, de biologistes ou microbiologistes pour les entreprises agrochimiques et de chercheurs ou chimistes pour l'ensemble des domaines cités. Nous partons de l'hypothèse que cette double spécialisation confère à ces personnes un statut d'intermédiaires entre le monde scientifique et le grand public. Sophie Moirand (2004: 4) a montré que les journalistes remplissent traditionnellement cette fonction, dans la mesure où ils «sont souvent considérés comme des médiateurs, des intermédiaires dont le rôle est de transmettre les connaissances issues des communautés spécialisées au grand public » (Peynaud $2014: 22$ ).

9 Leur double statut garantit aux spécialistes une crédibilité accrue, qui a toute son importance dans le processus de médiation des connaissances. Cependant, leur fonction ne se limite pas à la transmission d'informations à contenu scientifique ou technique : en tant que membres de l'entreprise, leurs propos servent aussi de relais au discours des multinationales auxquels ils appartiennent. Il s'agit de faire comprendre le 
contexte de production de leurs activités, ainsi que la manière dont ils envisagent leur rôle au sein de leur institution et, plus largement, de la société. En ce sens, nous souhaiterions montrer que les spécialistes d'entreprise produisent un «discours de médiation » sur la science et la technologie, marqué par les visées communicationnelles de leur entreprise d'appartenance. C'est la raison pour laquelle nous privilégions le terme "médiation" (plutôt que "vulgarisation»), qui permet d'adapter le degré de spécialisation en fonction de l'importance de la controverse. Ce contexte particulier pose bien entendu la question de l'indépendance des spécialistes et de leur liberté d'expression lorsqu'ils abordent des sujets controversés.

\section{Les spécialistes d'entreprises, des ambassadeurs indispensables du milieu spécialisé}

\subsection{L'évolution des pages d'accueil des entreprises}

Les résultats de l'analyse diachronique des pages d'accueil des sites Internet des entreprises entre 2000 et 2015 montrent une évolution dans la caractérisation de l'identité de l'entreprise et de ses acteurs. On remarque ainsi le glissement d'un contenu typique du discours de l'entreprise, peu personnalisé et centré sur des informations financières ou promotionnelles (sections Products, News ou Investors, principalement), vers une forme de personnification de l'image de l'entreprise, identifiée à travers ses membres ou ses clients. Les pages les plus récentes se distinguent par une diversification des représentants de l'entreprise, à travers la présence quasi systématique de la figure du spécialiste ou du scientifique.

En 2002, les pages d'accueil de Merck et de Pfizer ne présentaient pas de référence notable au secteur pharmaceutique : chez Pfizer, seuls les onglets Health, Medicine and Lifestyle, The Science of Medicine et Caring for Pets and Livestock permettaient d'identifier l'activité de l'entreprise. En revanche, les autres éléments visuels et textuels de la page d'accueil correspondaient aux sections traditionnelles de la communication d'entreprise (Products, Newsroom, Financial Information/Investing in Pfizer, Careers). 
Fig. 1. Page d'accueil du site Internet de Merck (août 2002)

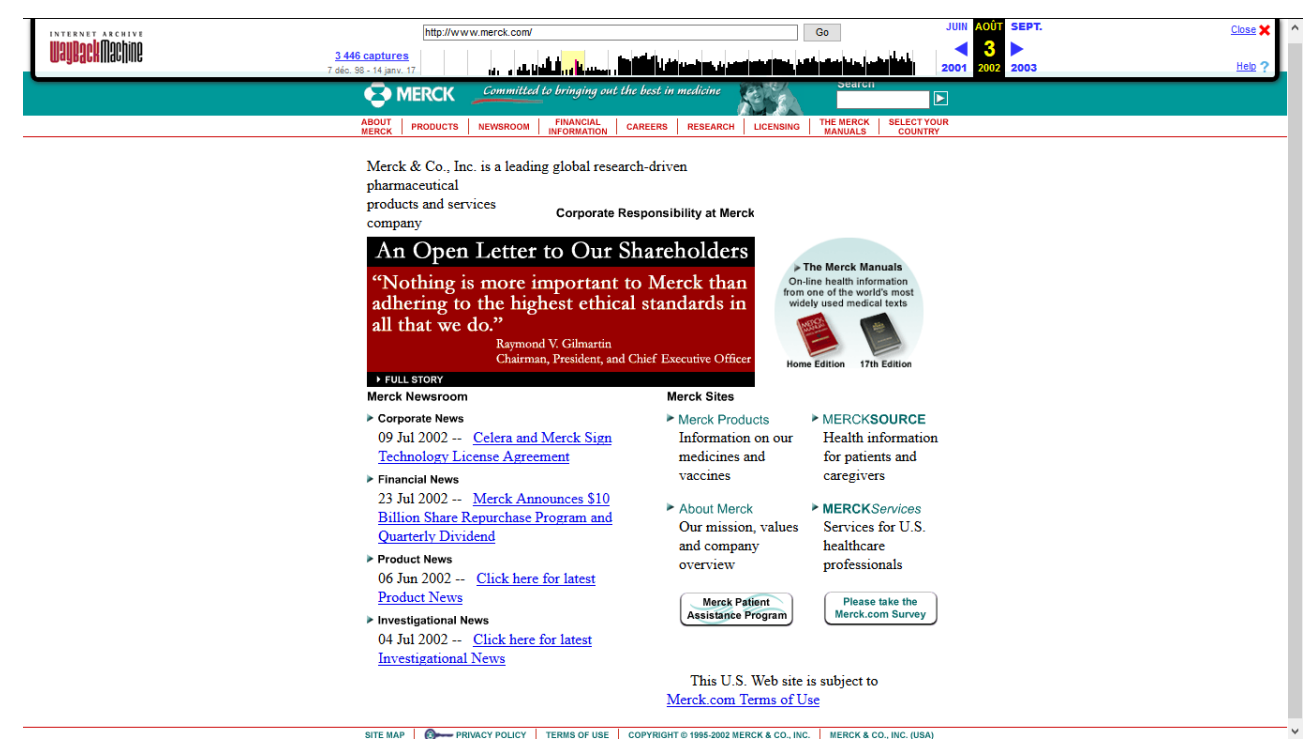

Fig. 2. Page d'accueil du site Internet de Pfizer (août 2002)

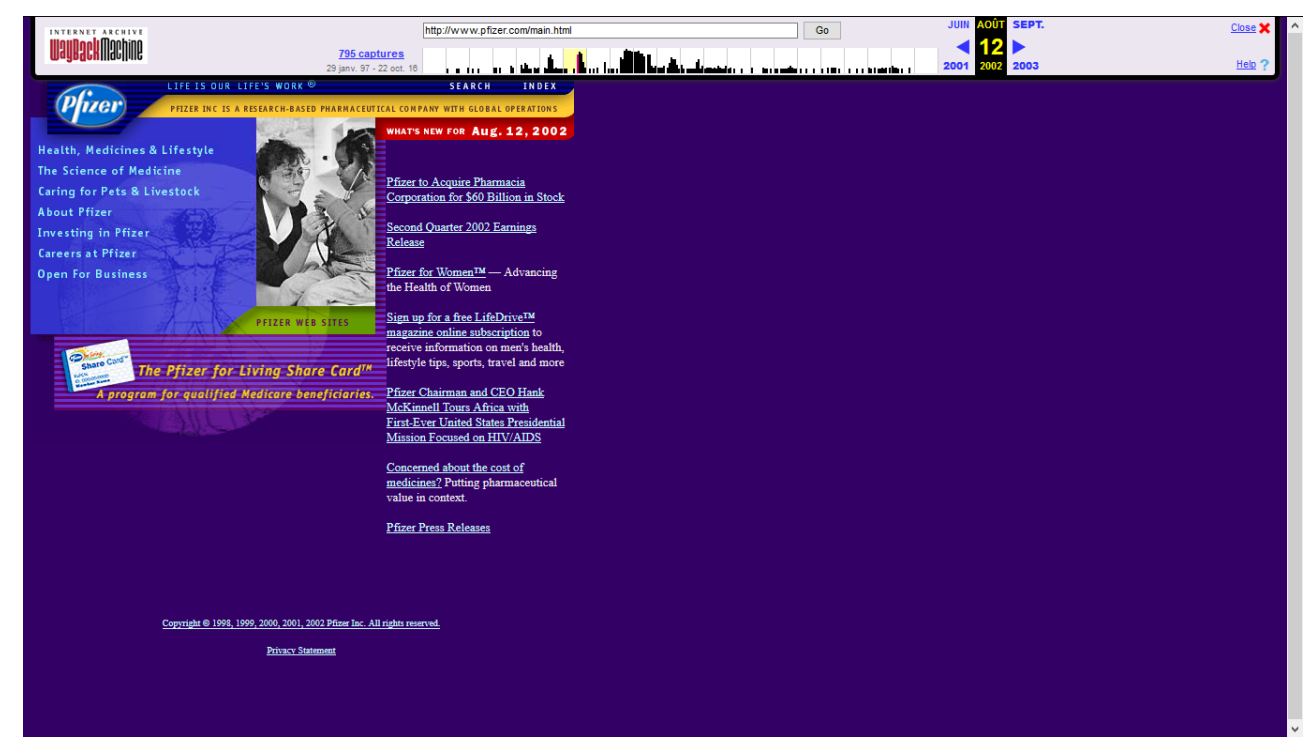

12 En novembre 2015 en revanche, la première image qui apparaît sur la page d'accueil du site de Merck est celle d'un scientifique en pleine action, liant explicitement l'identité de l'entreprise au monde de la recherche. Le choix d'une image en noir et blanc et la référence à trente ans d'engagement dans la recherche contre le sida soulignent l'importance historique de la recherche pour les activités de l'entreprise. 
Fig. 3. Page d'accueil du site Internet de Merck (novembre 2015)

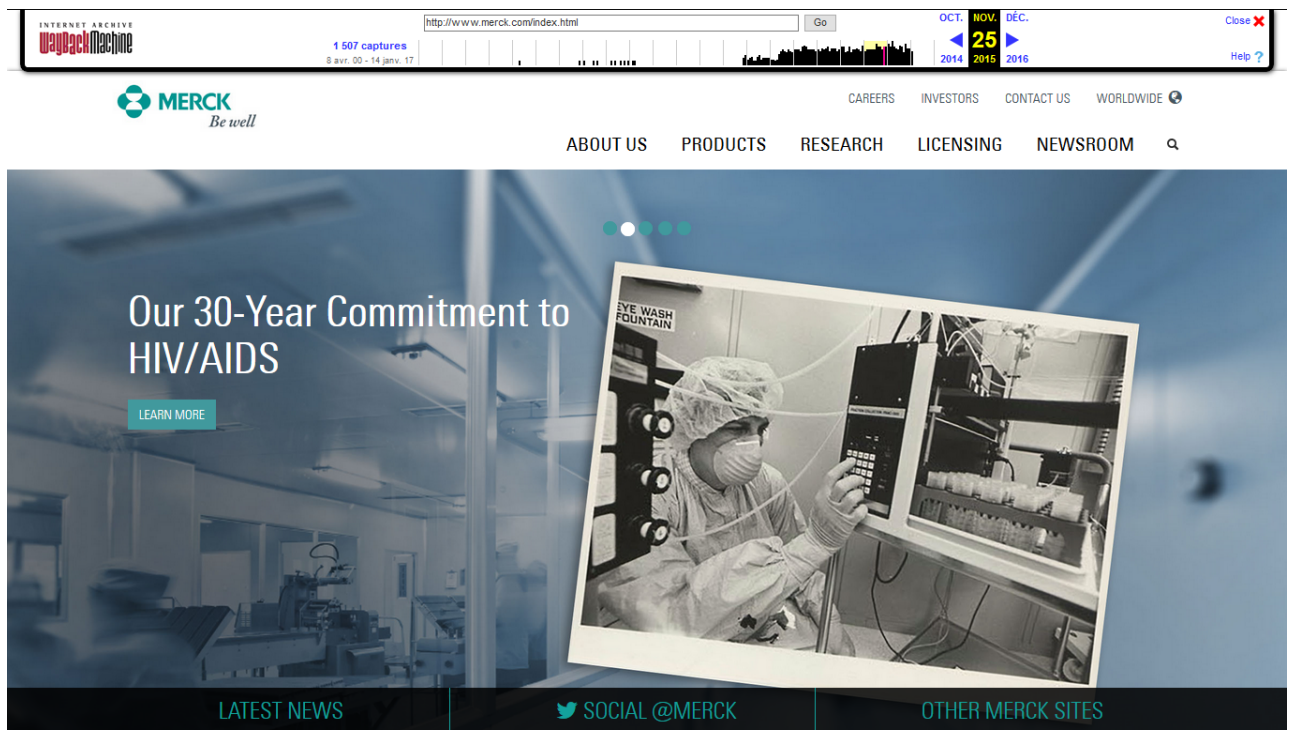

En écho à Merck, la page d'accueil du site de Pfizer met également en scène un scientifique. Ici, le gros plan, les éléments flous au premier plan, le cadrage resserré et l'air affairé de l'expert semblent mettre l'accent sur l'urgence des problèmes sanitaires auxquels répondent les produits de l'entreprise.

Fig. 4. Page d'accueil du site de Pfizer (août 2016)

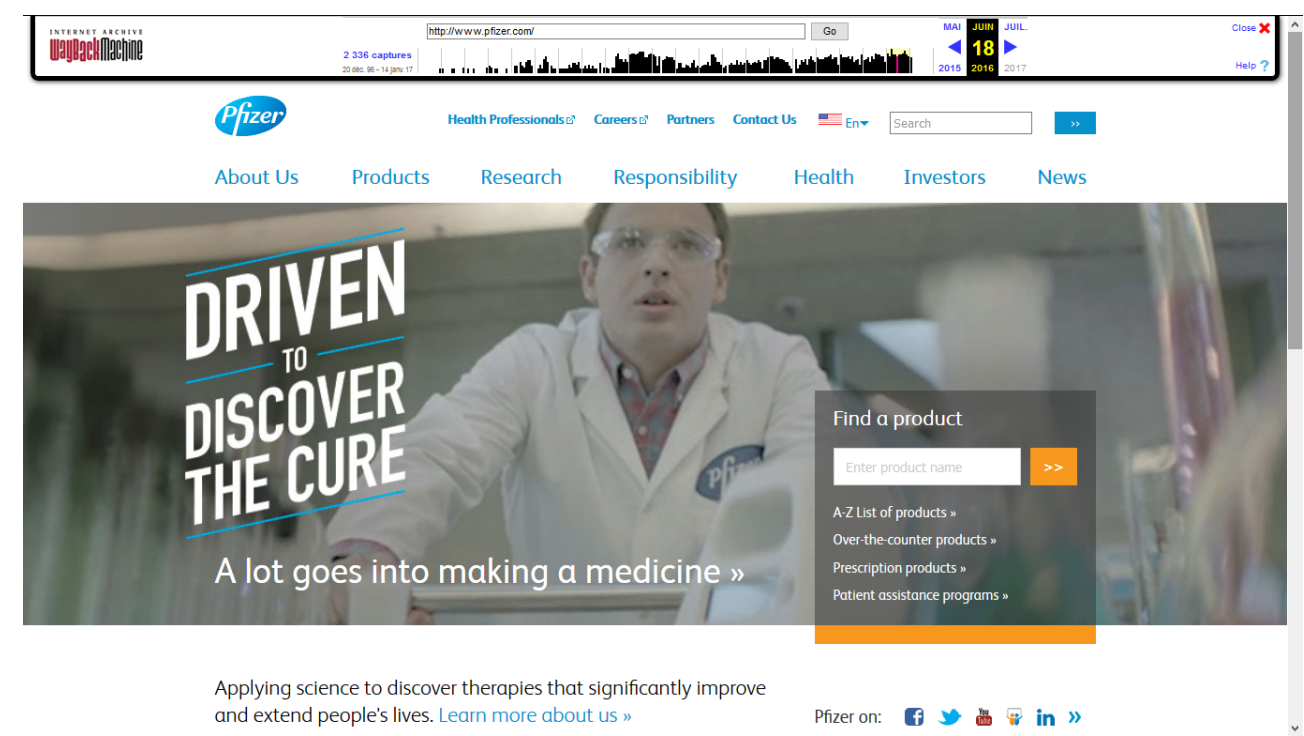

14 À l'image de Merck, la page d'accueil de Pfizer contient une référence au champ lexical de l'engagement («driven to discover the cure»). On peut penser que les photos de scientifiques qui consacrent toute leur énergie à la découverte de nouveaux médicaments visent à promouvoir l'image d'entreprises soucieuses de leurs patients, plutôt que de leur propre profit. La systématisation de l'image du spécialiste contribue ainsi à une redéfinition des activités et des valeurs de l'entreprise, conformément aux nouveaux enjeux liés à la citoyenneté d'entreprise : au-delà de la recherche de profit, les services de l'entreprise doivent également bénéficier à l'ensemble de la société (Valor 2005 : 193). 
De fait, cette idée d'un «bénéfice mutuel » (ibid.) était déjà présente sur les sites les plus anciens des entreprises agrochimiques: les pages d'accueil publiées en 2000 mettaient en avant des photos de producteurs ou de cultures, associant les enjeux de l'entreprise et ceux du monde agricole. En écho à ce qui a été observé dans le secteur pharmaceutique, les références au domaine de la science restaient anecdotiques au début des années 2000 (des onglets consacrés aux biotechnologies et une référence au Green Chemistry Challenge chez Dow AgroSciences).

Fig. 5. Page d'accueil du site Internet de Dow AgroSciences (octobre 2000)

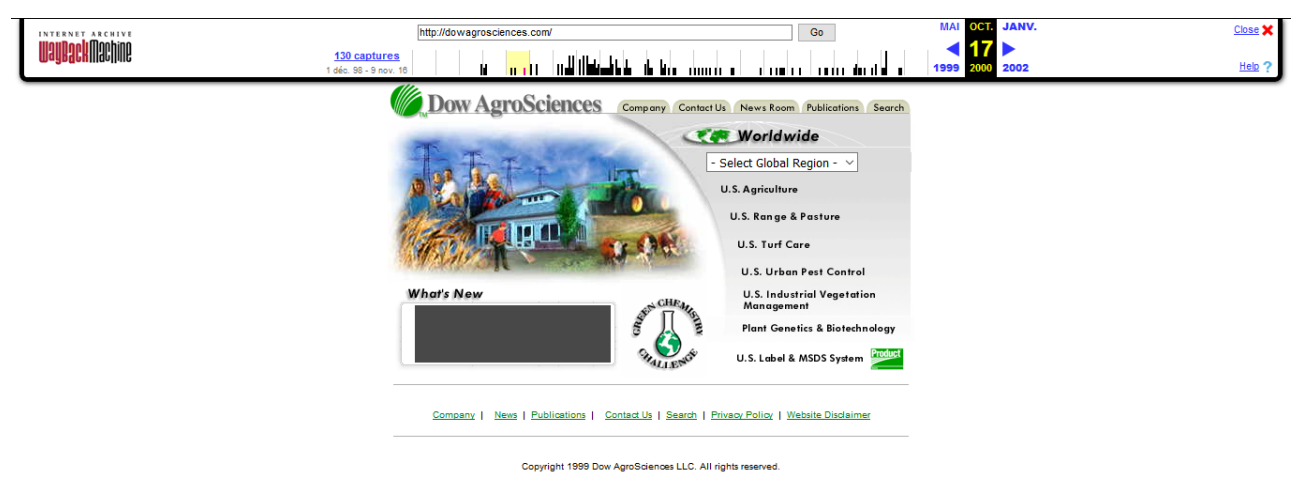

Fig. 6. Page d'accueil du site Internet de Monsanto (janvier 2001)

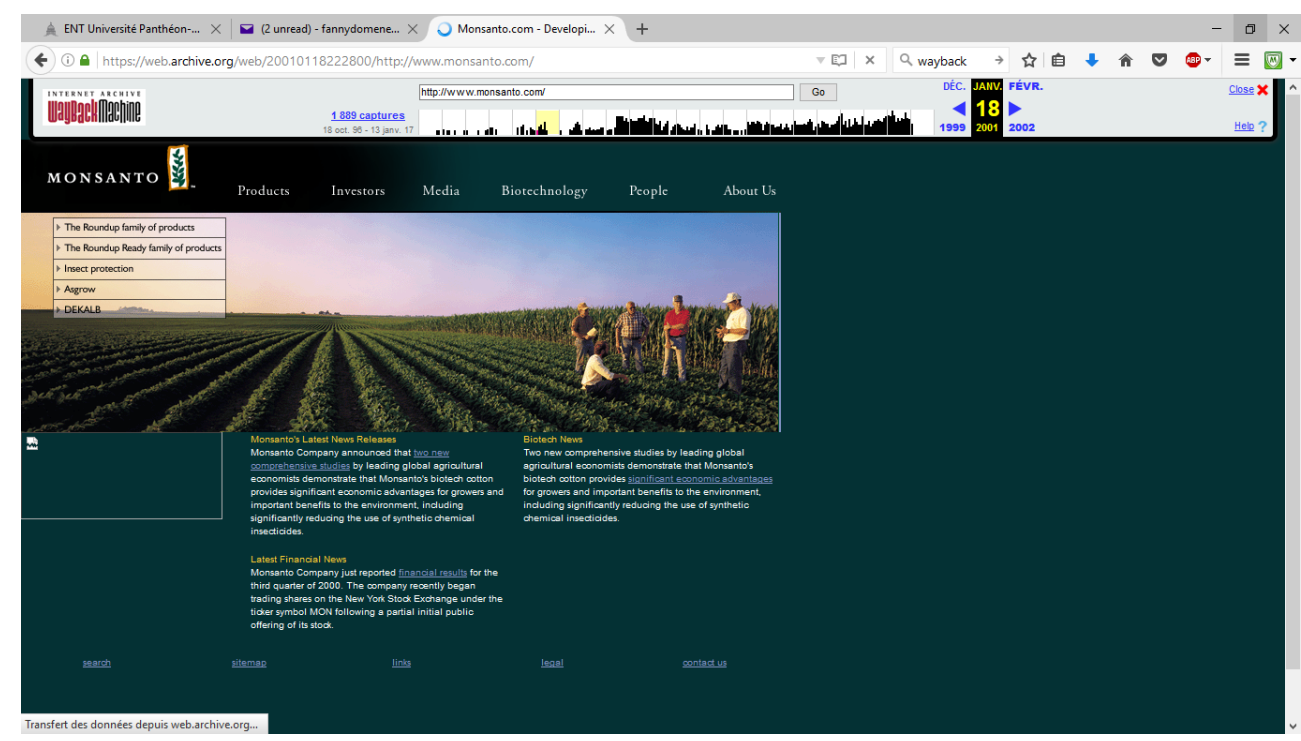

Cependant, en novembre 2015, c'est une scientifique qui est choisie pour représenter Dow AgroSciences, sur l'une des quatre illustrations qui se succèdent sur la page d'accueil. La légende de la photo ("Our pipeline is the best and most diverse in history") souligne la diversité des recherches menées par les chercheurs, et suggère l'importance croissante de la communauté scientifique parmi les membres de l'entreprise. 
Fig. 7. Page d'accueil du site Internet de Dow AgroSciences (novembre 2015)

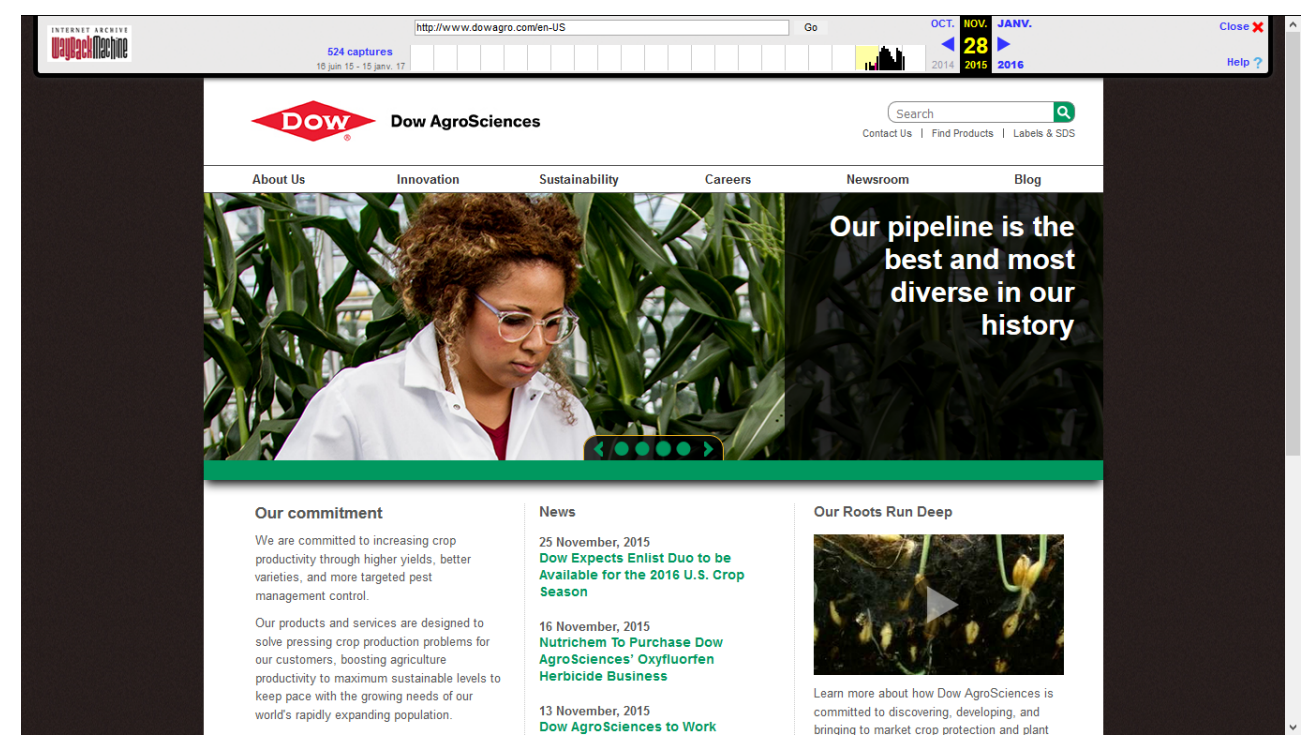

17 Sur la page d'accueil de Monsanto, le monde agricole et les producteurs restent omniprésents. Cependant, en écho à ce qui a été identifié chez Dow AgroSciences, la section Where the Jobs Are accessible depuis la page d'accueil met exclusivement en avant des collaborateurs scientifiques ou des ingénieurs.

Fig. 8. Page d'accueil du site de Monsanto (septembre 2016)

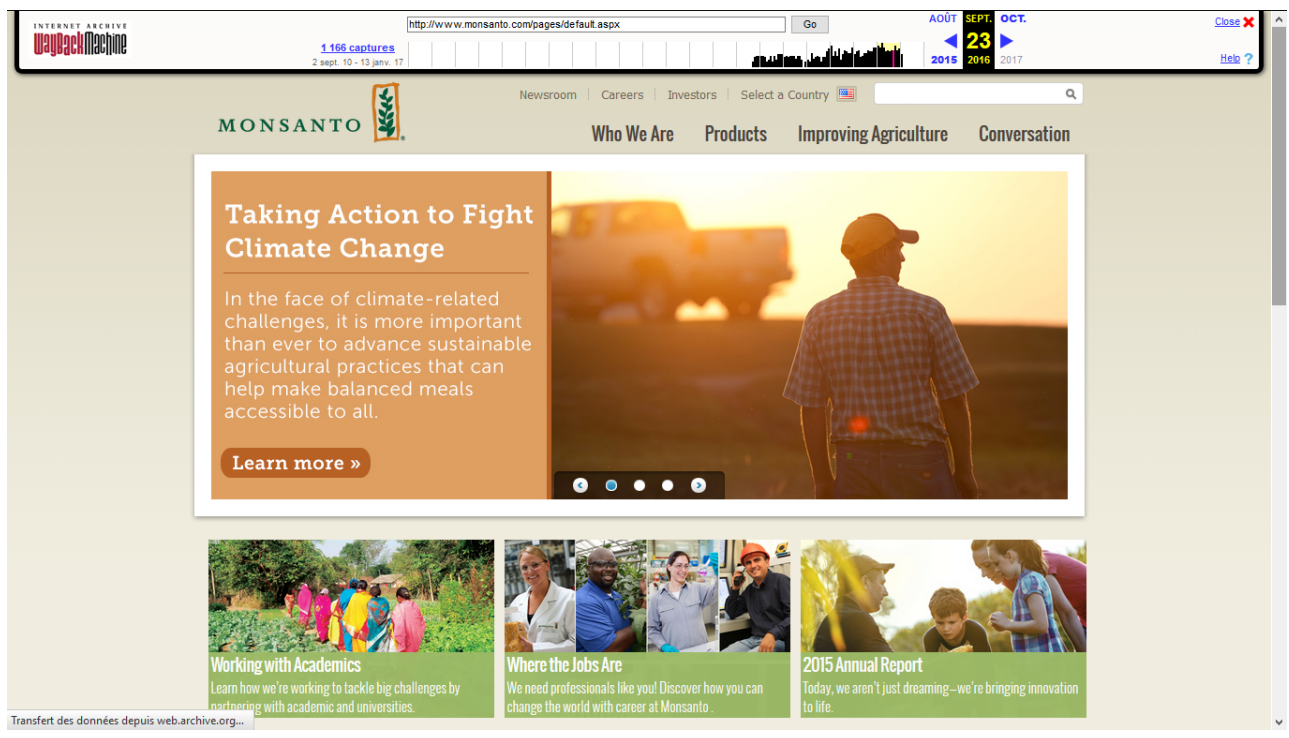

Dans le secteur de l'énergie, ce sont les ingénieurs qui sont choisis pour promouvoir les performances techniques (Chevron) ou environnementales (ExxonMobil) de l'entreprise sur leurs pages d'accueil.

Fig. 9. Page d'accueil du site Internet de Chevron (mai 2015) 


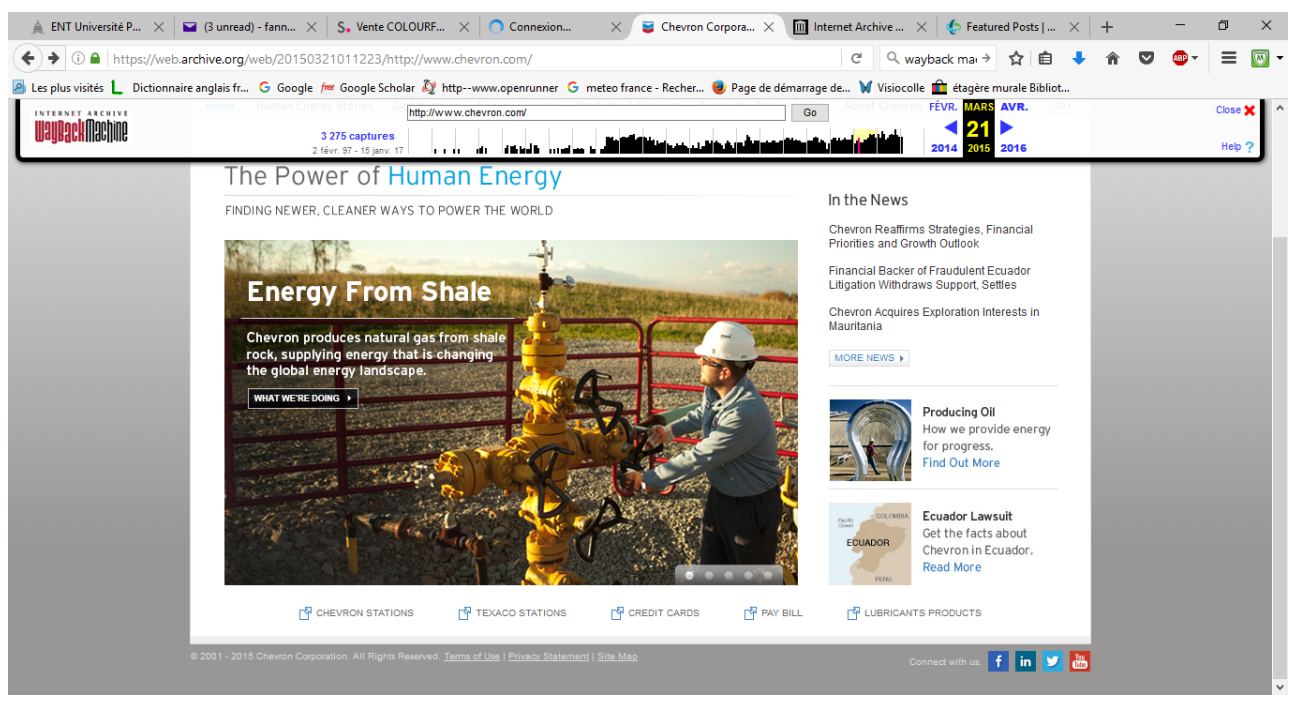

Fig. 10. Page d'accueil du site Internet d'ExxonMobil (novembre 2015)

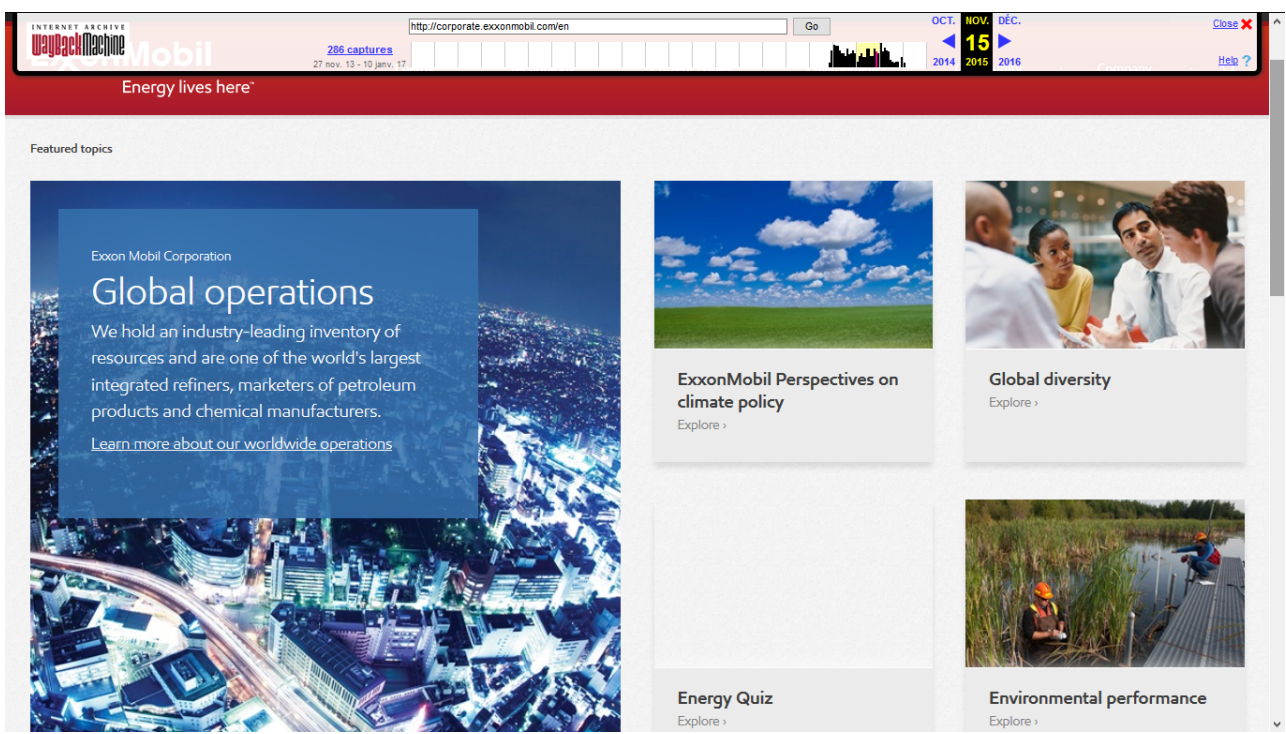

Il convient de noter ici que les pages d'accueil les plus récentes de Duke Energy et d'Entergy incluent très peu d'éléments visuels et que ces derniers sont sans rapport avec les membres de l'entreprise. En revanche, les pages About Us des deux entreprises incluent la photo d'une femme ingénieur (Duke Energy) ou d'un groupe d'ingénieurs (Entergy), qui illustrent la mission de l'entreprise : 
Fig. 11. Page About Us Duke Energy (novembre 2015)

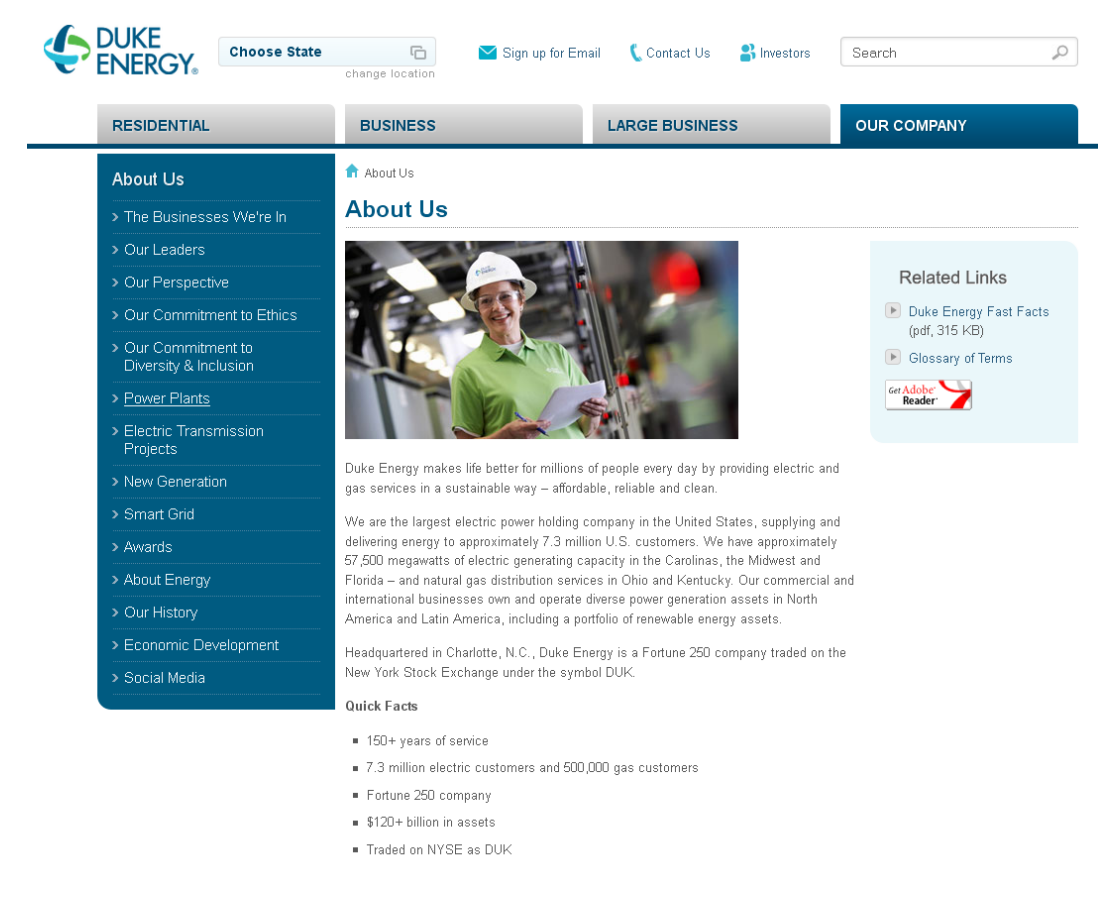

L'analyse du contenu visuel et textuel des pages d'accueil montre que les scientifiques et les ingénieurs sont devenus des ambassadeurs indispensables du milieu spécialisé, en lieu et place d'autres acteurs traditionnellement liés au monde de l'entreprise (président directeur général, responsable communications, responsable juridique, responsable marketing). On remarque également que, sur les images, les spécialistes présentent les attributs traditionnellement associés au scientifique ou à l'ingénieur : blouse blanche, masque et lunettes de protection ou casque. On peut penser que ces différents éléments visent à renforcer l'image d'entreprises responsables, en mettant l'accent sur les impératifs de sécurité propres au domaine du risque technologique.

Dans la mesure où les genres traditionnels du discours de l'entreprise ne sont $a$ priori pas destinés à relayer un discours incluant du contenu scientifique, la diversification des représentants du milieu spécialisé va de pair avec une diversification des canaux de communication mis en place par les entreprises. Ainsi, les critères prototypiques des rapports annuels ou des communiqués de presse des multinationales du pétrole et de l'agrochimie ont évolué pour inclure des citations ou des témoignages de scientifiques internes à l'entreprise (Domenec 2013). Sur les sites Internet des entreprises étudiées, différentes sections consacrées aux spécialistes proposent d'en apprendre plus sur leur formation ou sur la façon dont ils envisagent leurs fonctions au sein de l'entreprise.

\subsection{Décrire la mission des spécialistes : des outils de communication originaux au sein du discours de l'entreprise}

22 On distingue deux catégories de pages Internet consacrées aux spécialistes : d'une part, des entretiens filmés ou des comptes rendus d'entretien, où les spécialistes partagent 
leur expérience personnelle et professionnelle et, d'autre part, des vidéos qui présentent les travaux des ingénieurs et des scientifiques employés par l'entreprise.

En écho à ce qui a été identifié sur les pages d'accueil, la mise en image des scientifiques et les titres choisis pour les entretiens participent d'une forme de personnification de l'entreprise. Ce procédé de personnification peut être explicite (Humans for Health, Merck, Powered by Our People, Duke et Entergy sur le site du Nuclear Energy Institute) ou implicite, lorque l'utilisateur est directement invité à rencontrer les scientifiques qui font l'entreprise (Meet our Scientists, Pfizer). La mention du nom des spécialistes permet de créer une proximité avec l'utilisateur, qui contraste avec l'idée d'une entité anonyme et déshumanisée.

Fig. 12. Page Meet our scientists, Pfizer (novembre 2015)

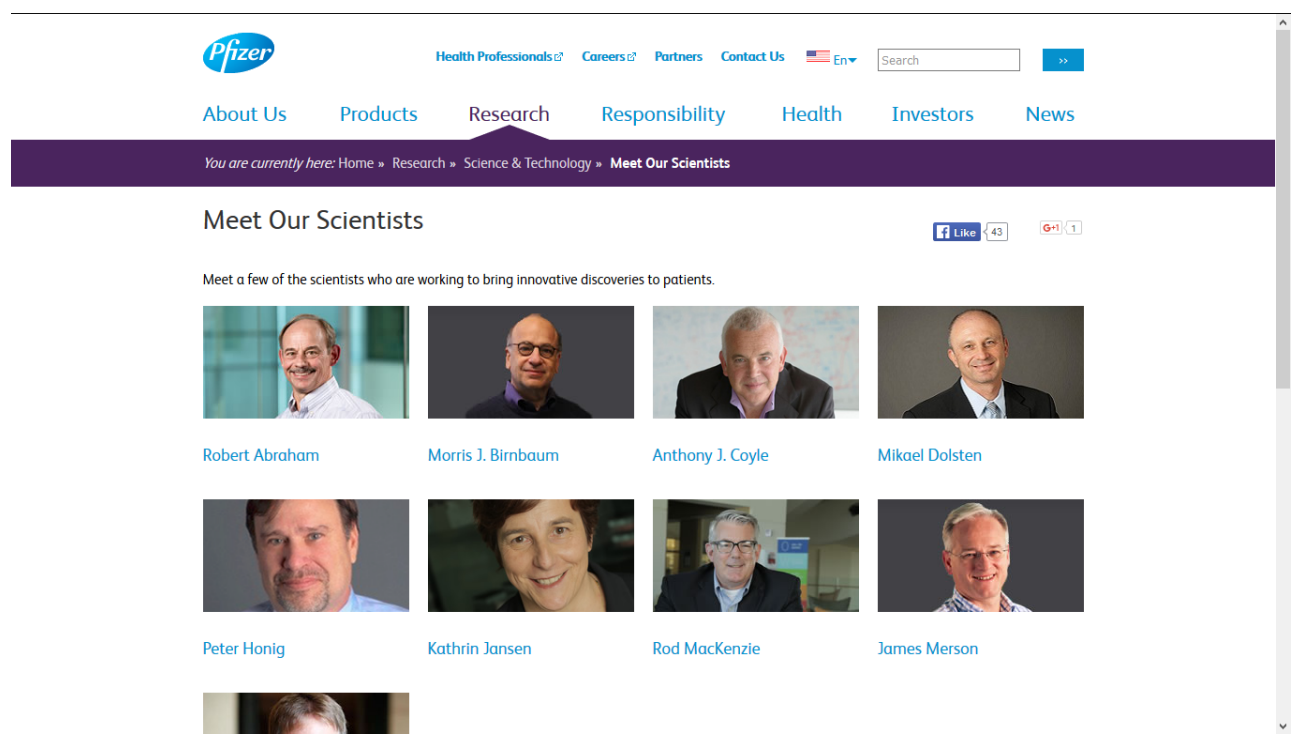

Fig. 13. Page Featured Scientist, Dow AgroSciences (novembre 2015)

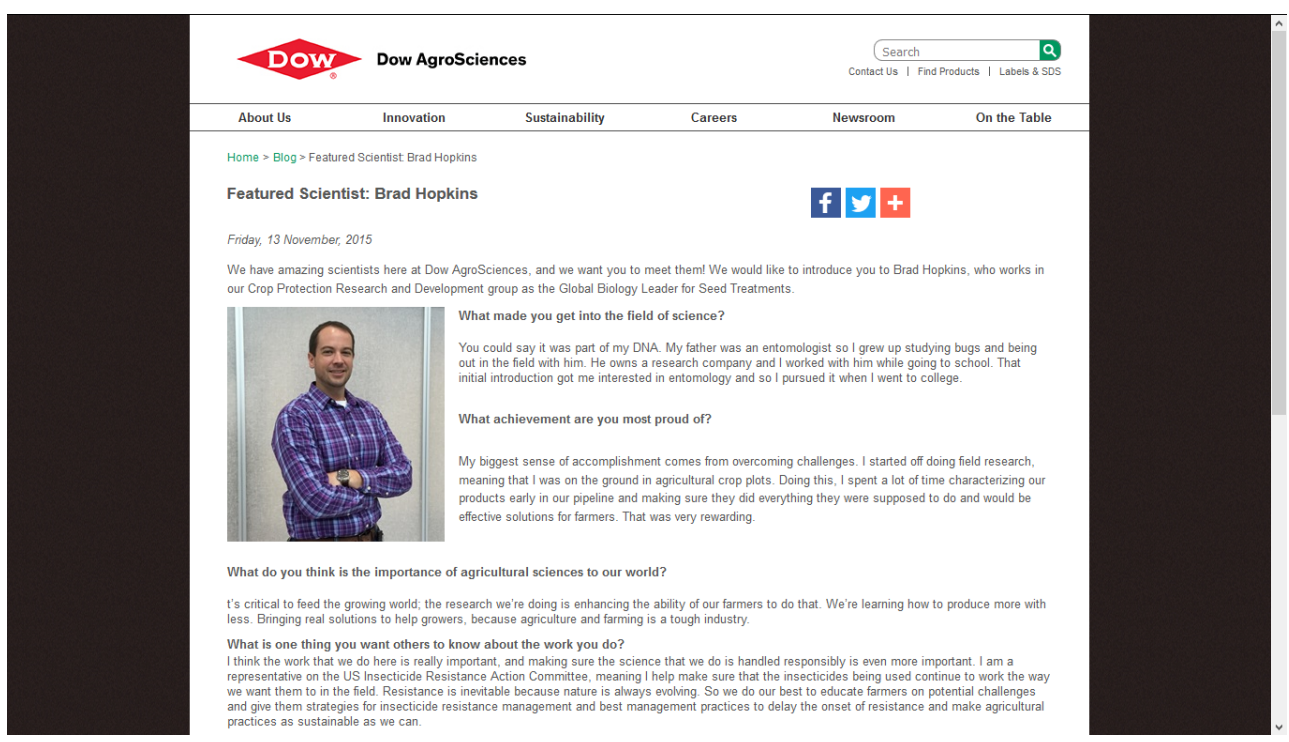


Dans les entretiens, l'identité des spécialistes est avant tout liée à leur statut de scientifiques plutôt qu'à leur fonction au sein de l'entreprise (ajout des mentions $M D$, Ph.D. ou de leur champ d'expertise).

Fig. 14. Page Humans for Health, Merck (novembre 2015)

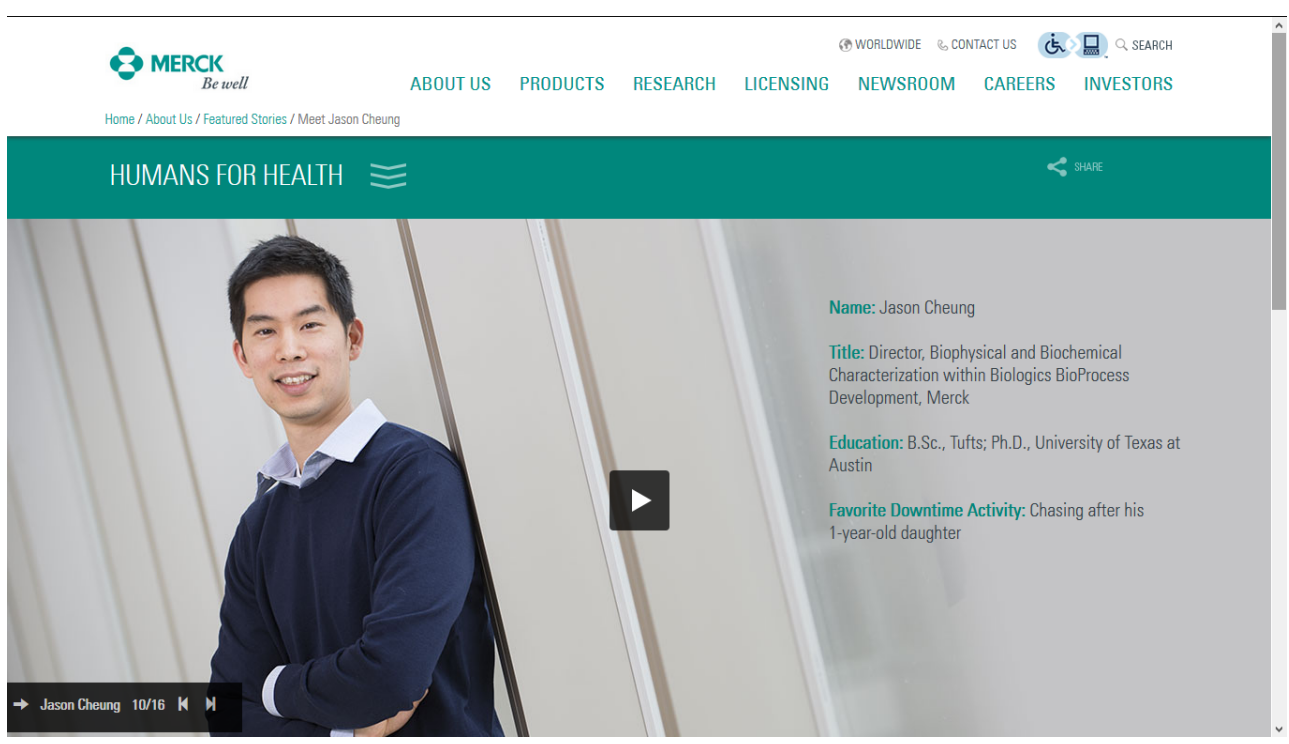

Cependant, contrairement à ce qui a été observé sur les pages d'accueil, les attributs caractéristiques du scientifique ont disparu dans ces sections. Il semble que l'expert est présenté comme un membre de l'entreprise parmi les autres. En ce sens, il s'agit également d'humaniser le processus technique et scientifique, mené par des hommes et des femmes auxquels le public peut s'identifier. Loin de l'image négative de l'apprenti sorcier ou du savant fou, les scientifiques mentionnent des souvenirs d'enfance ou des activités simples, partagées par le plus grand nombre :

Merck [1]: Humans for Health

Jason Cheung. Favorite activity: Chasing after his 1-year-old daughter

Ayesha Sitlani, Ph.D. Favorite activity: Watching "Downton Abbey"

Dow AgroSciences [1]: Featured scientist

What made you get into the field of science?

Brad Hopkins: You could say it was part of my DNA. My father was an entomologist

so I grew up studying bugs and being out in the field with him.

Entergy [1]: Nuclear Energy Institute

Tiffany Williams, security operations supervisor at Entergy ${ }^{4}$

I owe my love of nuclear power to my father. As a child, I remember him coming

home from work and sharing stories of what he did that day. We were fascinated because it was like nothing else we heard before.

De fait, les entretiens permettent également aux spécialistes d'expliquer leur conception de leur métier et des progrès permis par la science :

Merck [2]: Humans for Health

Daria Hazuda, Ph.D. We want to make a difference. We're always driven to push the frontier, to do something that nobody has ever done before. That's why we've become scientists.

Pfizer [1]: Meet our scientists

Robert Abraham. We are now entering an era in which we can realistically expect to develop therapies that induce very durable or even curative responses in patients afflicted with cancer that were previously considered incurable. 
Dow AgroSciences [2]: Featured scientist

Natalie Giampietro. The field of science is based on asking questions and searching for answers. As I looked at skills needed for different jobs, I discovered that a career as an organic chemist would provide a channel to fulfill my curiosity.

Dans le domaine du nucléaire, le site du Nuclear Energy Institute ${ }^{5}$ inclut une section Powered by our People, qui regroupe des vidéos et des billets postés par des spécialistes de différentes multinationales. Comme dans les entretiens publiés sur les sites des entreprises pharmaceutiques et agrochimiques, les ingénieurs et les chercheurs insistent sur les raisons pour lesquelles ils ont choisi le domaine du nucléaire :

Entergy [2]: Nuclear Energy Institute

Tiffany Williams, security operations supervisor at Entergy ${ }^{6}$

I'm proud to be a second generation nuclear worker. Just like my father, I will continue to educate people about the safe, reliable and clean air energy generated from nuclear. It's important for America's energy future.

Chez Chevron, une catégorie de spécialistes appelés les Fellows, regroupe ceux dont les contributions sont « visionnaires»:

Chevron [1]: Chevron Fellows ${ }^{7}$

Delivering energy to the world requires a wide range of technologies and the best and brightest minds to develop them. At Chevron, we employ thousands of technologists, but membership in the Chevron Fellows is reserved for those whose contributions are best described as visionary.

La vidéo qui leur est consacrée mêle plusieurs témoignages (voir figure 15).

Fig. 15. Page Chevron Fellows, Chevron (novembre 2015)

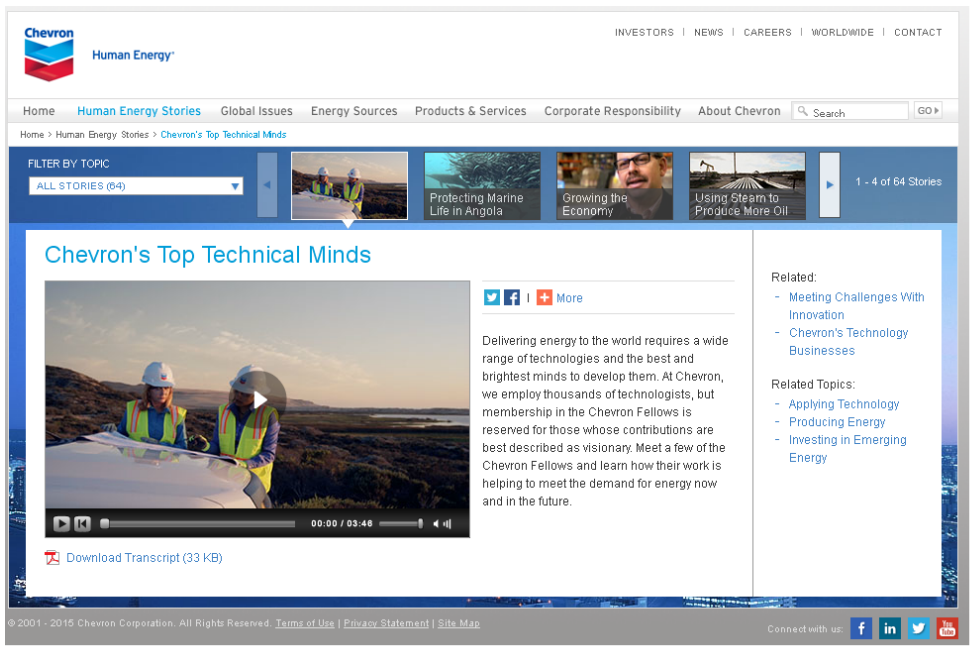

Contrairement aux entretiens individuels privilégiés pour présenter les spécialistes des entreprises pharmaceutiques et de Monsanto, les vidéos consacrées aux Chevron Fellows 
soulignent la diversité des champs d'expertise représentés chez cette catégorie de spécialistes :

Chevron [2] ${ }^{8}$

Harry Sigworth, Mechanical Engineer and Chevron Fellow

We come from many different backgrounds, but we're the same in that we do a

good job of applying technology.

31 Au-delà des témoignages des spécialistes, la vidéo constitue un canal privilégié pour illustrer les expériences conduites au sein de l'entreprise et les rendre plus accessibles au grand public. Sur le site de Monsanto, deux vidéos intitulées Monsanto's Breeding Efforts I et Monsanto's Breeding Efforts II décrivent les différents procédés mis en œuvre par les chercheurs de l'entreprise pour sélectionner la meilleure variété de semences. Les différentes séquences de la vidéo permettent de clarifier l'utilisation des marqueurs moléculaires ou d'une technique appelée seed chipping, qui consiste à prélever une fine pellicule de la semence étudiée pour déterminer sa composition génétique (voir figure 16).

Fig. 16. Vidéo Monsanto's Breeding Efforts II, Monsanto (novembre 2015) : marqueurs moléculaires

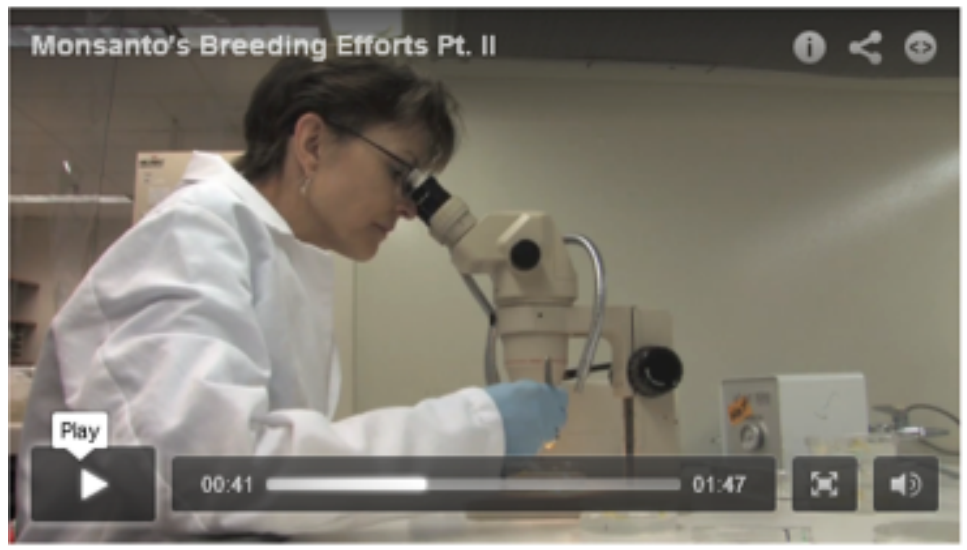

Fig. 17. Vidéo Monsanto's Breeding Efforts II, Monsanto (novembre 2015) : technologie dite du seed chipping

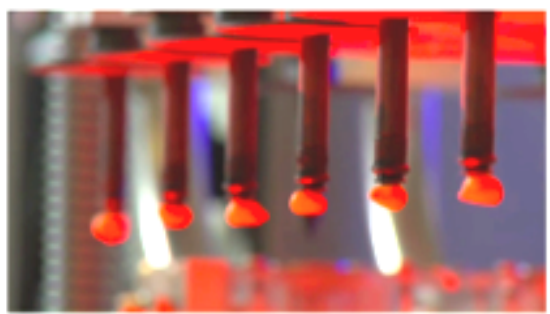

Les exemples sélectionnés montrent que la description de leur mission par les spécialistes du domaine présente un gradient de spécialisation, qui va du moins spécialisé (souvenirs d'enfance, détails de la vie quotidienne) à un haut degré de technicité (description d'expériences ou explications techniques). Les nouvelles 
fonctionnalités d'Internet permettent désormais aux entreprises de créer des médias spécifiques, afin d'inclure un discours technique ou scientifique au sein de la communication institutionnelle : au-delà des entretiens avec les spécialistes, un certain nombre de genres émergents spécifiques aux entreprises du risque technologique ont été identifiés.

\section{Les spécialistes comme médiateurs du discours scientifico-technique de l'entreprise}

\subsection{Expliquer la technologie pour légitimer les activités de l'entreprise}

Des recherches préliminaires ont permis d'identifier un argument-clé utilisé par les entreprises du pétrole et de l'agrochimie pour légitimer leurs activités : l'argument de l'acteur bienfaisant, selon lequel l'entreprise est au service de la société, notamment à travers la réponse aux besoins mondiaux (Kleinman \& Kloppenburg 1991). Sur les sites des entreprises pharmaceutiques, la plupart des scientifiques associent leur choix de travailler pour l'entreprise à la volonté d'être utiles à la communauté :

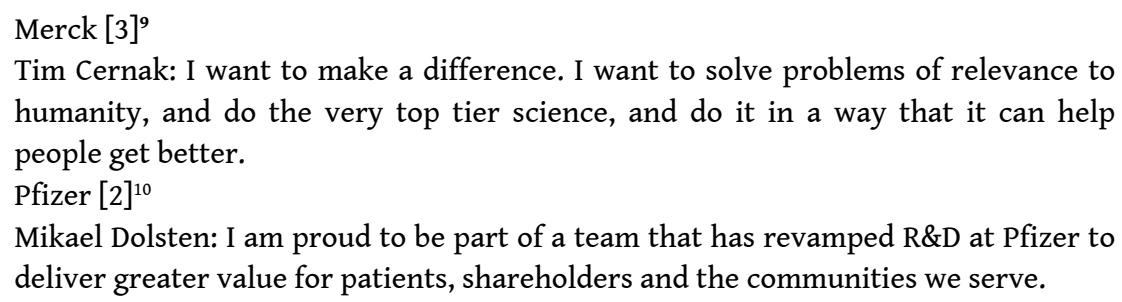

Dans la mesure où le principe même des médicaments est d'améliorer l'état de santé des patients, l'omniprésence du champ lexical lié aux solutions et à l'amélioration n'est pas surprenante. Cependant, on peut également y voir une réponse indirecte aux controverses fréquentes sur les prix trop élevés des traitements, ainsi qu'aux inquiétudes du grand public sur les effets secondaires potentiels des médicaments.

Ces inquiétudes sont d'autant plus fortes dans les domaines du pétrole et des biotechnologies végétales, où les produits chimiques utilisés ont un impact direct sur la santé et/ou sur l'environnement. Dans ces deux secteurs, les scientifiques décrivent leur mission dans des termes similaires à ceux de l'industrie pharmaceutique, en présentant l'activité de l'entreprise comme une réponse à des besoins mondiaux ou aux défis du futur:

ExxonMobil [1] ${ }^{11}$

Russell Tait (chief environmental scientist): Protect Tomorrow Today is a challenge as we look at the global range of our operations. Being able to assess how the environment will change naturally and in response to a range of potential developments from us and the rest of the community requires insight, a broad range of data and analytical skills.

Monsanto [1] $]^{12}$

Michael Clements: Monsanto scientists [...] can more precisely match the best potential parent plants to produce the best possible offspring that will be planted by farmers around the world.

Afin d'étayer l'argument de l'acteur bienfaisant, le discours des spécialistes sur les sites des entreprises étudiées vise également à présenter des technologies controversées 
comme le prolongement de phénomènes naturels (Kleinman \& Kloppenburg 1991), à travers des procédés de comparaison ou des métaphores.

Le procédé d'injection de vapeur d'eau (steam flooding) utilisé dans l'industrie pétrolière a été fortement critiqué pour son impact environnemental. Dans une vidéo consacrée à cette technique, deux ingénieurs de Chevron ont recours à des métaphores alimentaires pour la justifier. Le premier utilise l'image de la mélasse, obtenue à partir du raffinage du sucre, pour décrire l'aspect du pétrole, tandis que le second associe l'activité de l'entreprise à un procédé qui consiste à écrémer le lait :

Chevron $[3]^{13}$

Jeff Hatlen, Senior Petroleum Engineer, Chevron

The oil's thick and viscous. Think molasses. To get more of it out of the ground, we needed a new technology.

Chevron $[4]^{14}$

Jim Waldron, Hydrogeologist, Chevron

I think of Chevron at Kern River as a water company that skims oil.

Sur le site de Monsanto, les explications sur le rôle du scientifique dans le procédé de sélection génétique passent par la métaphore de la "rencontre arrangée », qui permettra d'envisager une naissance, même si le terme de "matchmaker » n'est plus ressenti consciemment comme métaphorique par bon nombre de spécialistes de phytogénétique. La métaphore filée, avec la référence à la descendance (offspring ou baby) paraît plus inoffensive et naturelle qu'une référence à des notions techniques comme la sélection ou la modification :

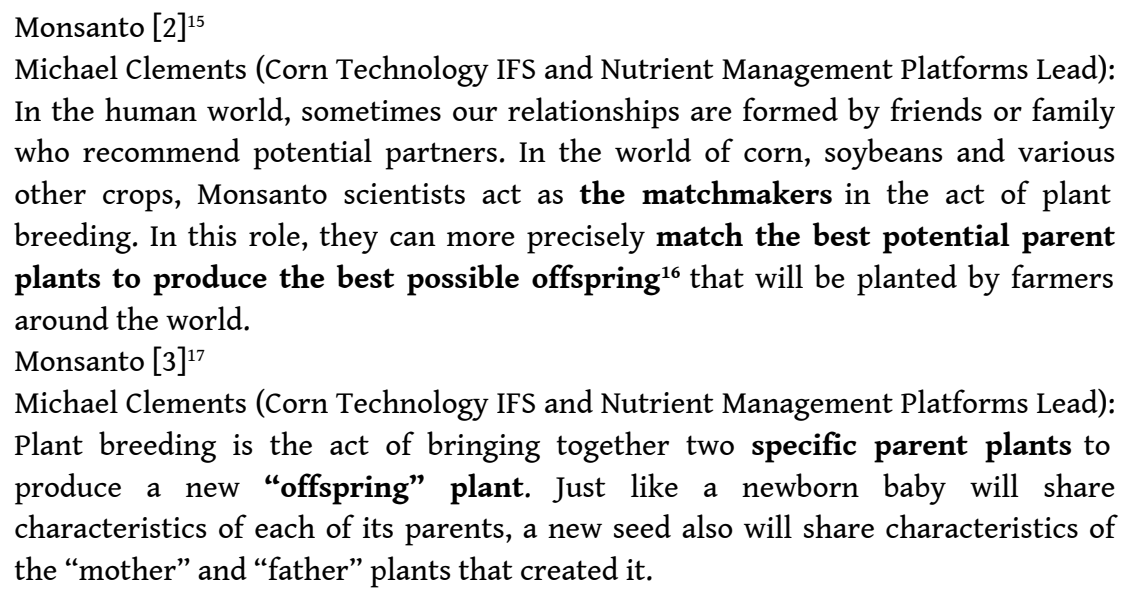

Sur le site de Pfizer, une vidéo explique la manière dont la médecine peut devenir plus écologique, à travers la mise en place des principes de la chimie verte : Green Chemistry at Pfizer: Want to Make Medicines Greener? It's in the Chemistry

Pfizer [3] ${ }^{18}$

When we talk about green chemistry, we talk about the design of efficient chemical processes that reduce or eliminate the use of hazardous materials.

[...] In our research divisions, we have developed solvent-selection tools to help scientists select greener solvents, reagents, acids and bases.

Ces exemples montrent que le scientifique en entreprise n'est pas nécessairement à l'origine d'un discours technique, fondé sur une terminologie précise. Ceci conforte notre conviction que le spécialisé ne se limite pas à un jargon propre à la communauté professionnelle ou au style spécialisé, mais s'appréhende en fonction du domaine et de ses enjeux communicationnels. 
41 Néanmoins, pour défendre des technologies fortement controversées, les entreprises ont mis en place des sites spécifiques sur lesquels les spécialistes répondent aux questions parfois très techniques $\mathrm{du}$ public. Ces nouveaux supports communicationnels, que nous avons regroupés sous l'appellation de "pagesplaidoyers » présentent une interaction avec le public.

\subsection{Les pages-plaidoyers : une médiation fondée sur une interaction avec le grand public}

La notion de "plaidoyer" a été empruntée à la responsable numérique de Dow AgroSciences, qui décrivait le site consacré au Chlorpyrifos, un insecticide fortement controversé, comme suit :

It's an advocacy site for a molecule that is I don't know how many years' old ... 50 years' old, and what we're doing is we're advocating for the continued use of

Chlorpyrifos. (Domenec 2013, entretien du 14 octobre 2011)

De fait, d'autres multinationales ont recours à des sites Internet spécifiques, afin de promouvoir des produits ou des activités controversés. En écho à des questions récurrentes du grand public sur l'impact environnemental et social de ses activités, Chevron a mis en place la campagne We Agree. Le principe est simple, diffuser des affirmations destinées à remporter l'adhésion du grand public: "It's time oil companies get behind the development of renewable energy. Protecting the planet is everyone's job ${ }^{19} »$.

Fig. 18. Page We Agree, Chevron (novembre 2015)

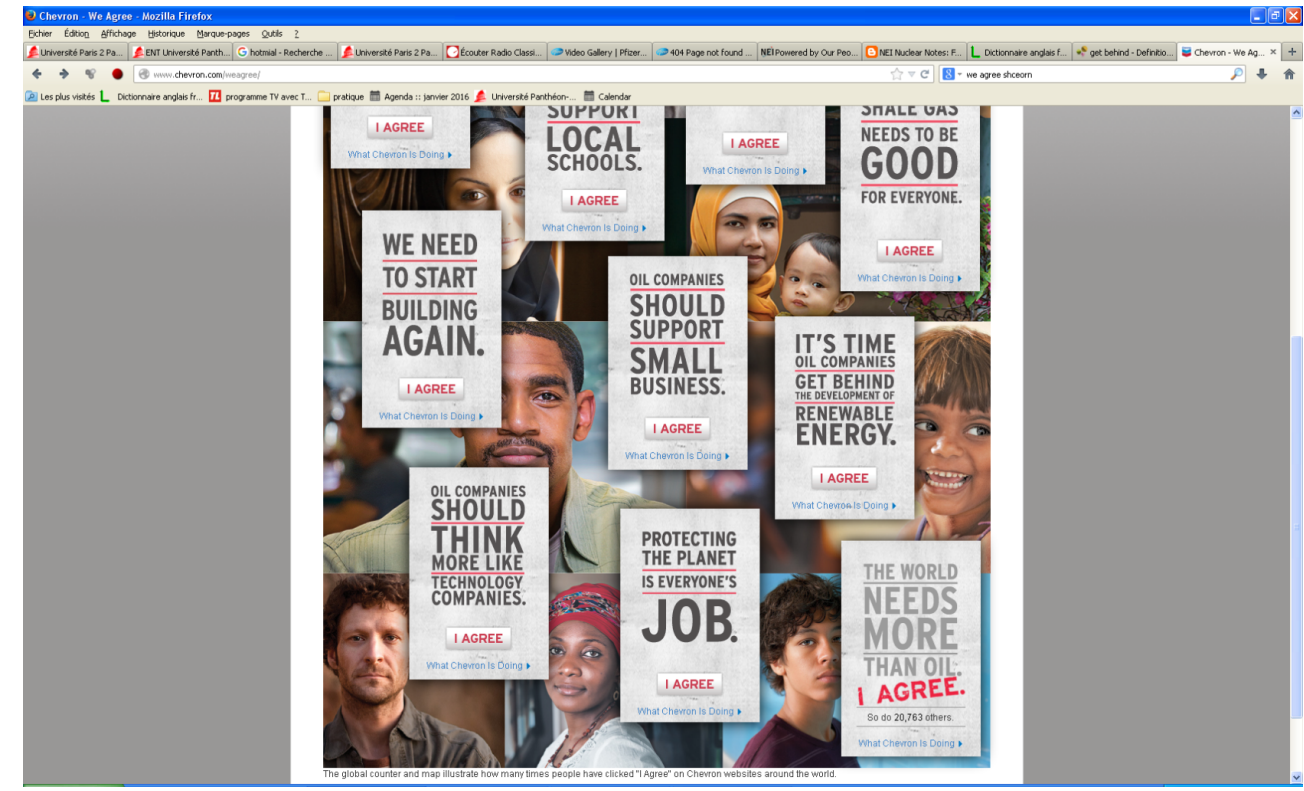

Les utilisateurs sont invités à cliquer s'ils sont d'accord avec cette affirmation et un lien intitulé What Chevron is doing les conduit à une page contenant des vidéos où des membres de l'entreprise expliquent ce que Chevron met en œuvre pour réaliser ces objectifs. 
En réponse au constat Shale gas needs to be good for everyone, des spécialistes employés par l'entreprise mettent en avant leur attachement à la terre où ont lieu les forages et leur volonté de protéger l'environnement :

Chevron $[5]^{20}$

Shawn Clark, Hydraulic Fracturing Specialist

Where the Marcellus is operating is where I wanted to be. I have family around here. I have friends. It's home to me. It's very important for us to protect this place. I mean, it's where we live.

Chevron $[6]^{21}$

Tim Berdar, Construction Team Lead

I've been here my whole life. I was born in Fayette County and don't want to leave Fayette County. I want to be here forever. As an avid hunter and fisherman I enjoy, you know, using the land and to my benefit and my family's benefit, generation after generation; so that's why I take responsibility in what we do. And the development of Marcellus Shale shows that we can do this - you know, extracting natural gas from the ground safely. And Chevron is a very, very safe company. You know, Chevron is going to set the bar for every shale operator in this area, there's no doubt.

L'utilisation de la première personne du singulier et le fait de s'adresser directement à l'utilisateur donnent l'impression d'une interaction avec l'expert. De plus, le recours à la première personne du pluriel (for us, we can do this) pour faire référence à Chevron participe de la stratégie d'humanisation de l'entreprise.

Plutôt qu'un site consacré à un produit ou à une activité, d'autres entreprises ont mis en place des blogs afin d'interagir avec le public. Sur le blog du Nuclear Information Center, des spécialistes de Duke Energy répondent aux questions du grand public sur différents aspects de l'énergie nucléaire.

Duke Energy [2] $]^{22}$

The Nuclear Information Center (NIC) provides an insider's look into nuclear energy. The NIC features insights into many aspects of the nuclear industry, including the benefits of nuclear energy, community events, emergency planning, videos and more. Content published here is written by specialists from Duke Energy's nuclear fleet.

Les billets portent sur différents aspects de l'énergie nucléaire, dont la santé et la sécurité, les mesures d'urgence mises en place par l'entreprise ou les mythes relatifs à l'industrie nucléaire (Health and Safety, Emergency Planning, Nuclear Myths), et ils peuvent être commentés par les utilisateurs. La fonction exacte des spécialistes n'est pas précisée, cependant, la photo d'une femme ingénieur sur la page d'accueil du blog suggère que ce sont des techniciens qui rédigent les billets et qui répondent aux questions des utilisateurs. 
Fig. 19. Page d'accueil du Nuclear Information Center (septembre 2016)
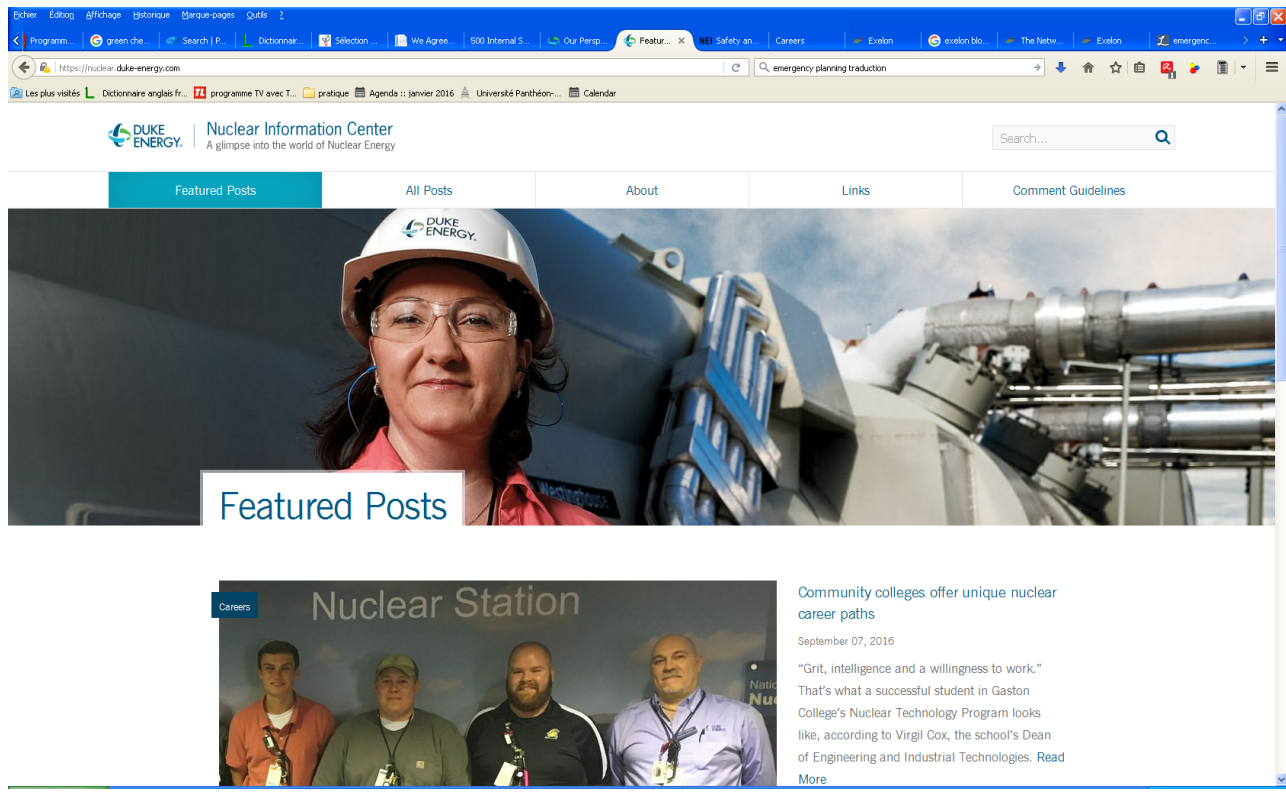

Les multinationales de l'agrochimie ont également développé des outils de communication spécifiques, où les spécialistes répondent aux questions du public : sur le site GMO Answers, l'utilisateur est assuré de trouver la meilleure réponse à sa question.

Fig. 20. Page d'accueil du site GMO answers (novembre 2015)

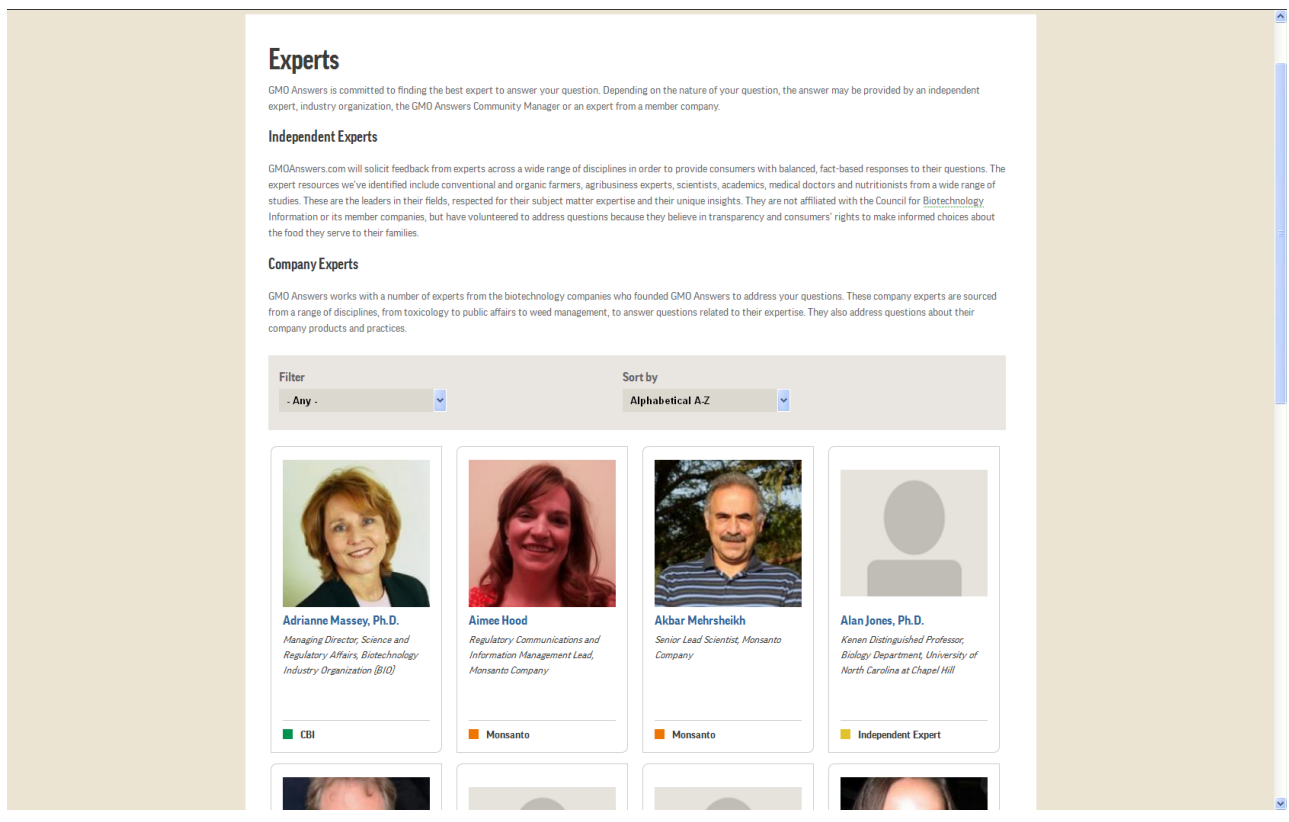

50 Le site présente certaines fonctionnalités typiques du blog, en permettant notamment une interaction avec les spécialistes. Selon l'information demandée, les questions sont transmises à différents interlocuteurs, choisis en fonction de leur expertise dans le domaine en question :

GMO Answers is committed to finding the best expert to answer your question. Depending on the nature of your question, the answer may be provided by an 
independent expert, industry organization, the GMO Answers Community Manager or an expert from a member company ${ }^{23}$.

51 Deux catégories de spécialistes sont mentionnées sur le site: les spécialistes indépendants (producteurs, universitaires, médecins ou nutritionnistes) et les spécialistes en interne. Une liste permet d'identifier les uns et les autres, en présentant leurs domaines de spécialisation respectifs, ainsi que l'organisation à laquelle ils appartiennent. À l'image du site du Nuclear Information Center, GMO Answers est présenté comme une interface entre la communauté scientifique et le grand public. Une question au sujet des effets du glyphosate, l'un des composants actifs du RoundUp, donne ainsi l'occasion à un scientifique de Monsanto de clarifier la distinction entre deux termes techniques :

Question Submitted By: brucemc from Wantagh, New York

Glyphosate bonds with lipids to create a highly reactive phospholipid analog of lecithin that crosses both the blood brain barrier and the placental barrier in higher lifeforms.

Expert Answer

By: Akbar Mehrsheikh, Senior Lead Scientist, Monsanto Company on Thursday, 10/15/2015 1:39 pm

Thank you for that scary sounding question. I wish you had said where you read that because there are lots of unfounded claims out on the internet and they are easiest to respond to with the details of the claim.

But this one is probably easy to answer since no such reaction, as described, has ever been noted or studied and it is inconsistent with the fundamental chemical properties of these molecules.

I can speculate, however, that the source of this confusion is simply a misunderstanding between the chemical terms "phosphonate" and "phosphate" 24

Au-delà de cette clarification terminologique, l'inclusion d'adjectifs évaluatifs comme scary-sounding, unfounded ou inconsistent semble destinée à discréditer les détracteurs du glyphosate, en présentant leurs critiques comme irrationnelles. Le statut de spécialiste d'Akbar Mehrsheikh lui confère plus de crédibilité que d'autres membres de l'entreprise (responsable communication ou chargé de relations médias), qui pourraient être accusés de ne pas maîtriser les aspects scientifiques et de prendre parti pour leur institution d'appartenance.

Les différents outils mis en place par les entreprises pour défendre des technologies controversées présentent un gradient de spécialisation, qui permet aux spécialistes de relayer un discours plus ou moins technique. Différents degrés d'interaction avec le public ont également été identifiés, allant de la simple communication d'informations (Chlorpyrifos, We Agree) à des supports favorisant l'interaction à travers des procédés de questions-réponses (GMO Answers) ou l'utilisation de blogs (Nuclear Energy Institute).

\section{Conclusion}

$54 \mathrm{Au}$ terme de cette étude, nous souhaitons revenir sur le statut particulier des spécialistes, qui occupent une place à part au sein du monde de l'entreprise et de la communauté scientifique. Leur double spécialisation, professionnelle et disciplinaire, leur confère un rôle décisif dans la communication d'entreprise. En tant que membres de l'organisation, ils bénéficient d'« un statut intermédiaire entre le monde scientifique et le grand public » (Peynaud 2014 : 23) et contribuent à la médiation du discours sur les nouvelles technologies, qui se distingue des procédés traditionnels de vulgarisation. La 
quasi-absence de terminologie ou de vocabulaire spécialisé confirme l'importance du domaine, plutôt que du lexique, comme facteur de spécialisation. D'autre part, la visibilité croissante des chercheurs, des médecins et des ingénieurs contribue à diversifier l'image des acteurs du milieu. Au-delà de la recherche de profit, traditionnellement considérée comme l'une des caractéristiques principales de l'entreprise (Friedman 1970 ; Colle $2008: 2$ ), les entreprises du risque technologique se présentent désormais comme des lieux de recherche et d'innovation, dont le but principal est d'améliorer le quotidien des membres de la société. L'analyse des pages d'accueil, sites, blogs et vidéos consacrés aux spécialistes en interne illustre un glissement progressif du discours des entreprises du pétrole, de l'agrochimie, du nucléaire ou de la pharmacie vers une forme originale de discours institutionnel centré sur la légitimation de l'innovation, plutôt que sur la promotion directe des activités de l'entreprise.

Cette stratégie de légitimation, ainsi que la mise en place de canaux de communication novateurs, semble confirmer l'hypothèse d'un domaine spécialisé du risque technologique caractérisé par des besoins communicationnels particuliers, au sein du monde de l'entreprise. Le risque technologique constituerait un facteur de spécialisation hybride, à la fois professionnel et disciplinaire, qui conditionnerait la culture du milieu professionnel et de ses acteurs. Il convient de noter ici que les sites GMO Answers et Nuclear Energy Institute sont caractéristiques d'une stratégie de " coopétition" (Nalebuff \& Brandenburger 1996 ${ }^{25}$ ), de plus en plus fréquente dans un contexte de controverse. Défini comme une «compétition coopérative " par John Humbley (2006: 97), le mot-valise "co-opétition » fait référence à «la combinaison paradoxale, simultanée de coopération et de compétition » (Yami et alii $2010: 6$ ) dans le monde de l'entreprise. Si le phénomène de co-opétition n'est pas limité aux entreprises du risque technologique, il semble que le besoin de légitimation propre aux secteurs du nucléaire ou de l'agrochimie favorise les initiatives de ce type au sein d'industries sujettes à controverse.

Dans la lignée de l'hypothèse d'un domaine spécialisé du risque technologique, de futurs travaux pourraient examiner d'autres exemples de cette stratégie de coopétition. Les discours produits par des scientifiques et des spécialistes internes à l'entreprise et des chercheurs universitaires pourraient également être analysés dans une perspective comparative, afin de mieux cerner les besoins communicationnels propres aux entreprises du risque technologique. Enfin, une interaction directe avec des spécialistes employés par l'entreprise pourrait permettre de clarifier le contexte de production de leur discours. On pourrait par exemple s'interroger sur le processus de sélection des spécialistes qui apparaissent et qui s'expriment sur les sites des multinationales étudiées ou sur d'éventuelles contraintes imposées par les entreprises. Il serait intéressant de savoir si les multinationales ont une influence sur le discours généré par leurs scientifiques et par les spécialistes, et dans quelle mesure certains choix thématiques ou terminologiques sont conditionnés par le statut de membre de l'entreprise. 


\section{BIBLIOGRAPHIE}

BEITONE, Alain et alii. 2010. Dictionnaire des Sciences Économiques. Paris : Armand Colin.

BENNET, Nathan \& James LEMOINE. 2014. « What VUCA really means for you ». Harvard Business Review 92/1-2, 27.

COLLE, David. 2008. Un monde d'entreprises. Paris : Presses Universitaires de France.

DOMENEC, FANNY. 2013. «Contribution à l'analyse du milieu spécialisé des entreprises du risque technologique à travers leur discours : le cas du pétrole et des biotechnologies agricoles aux États-Unis ». Thèse de doctorat en études anglaises, Université Paris Sorbonne.

FRIEDMAN, Milton. 1970. « The social responsibility of business is to increase its profits ». The New York Times Magazine, 13/09/1970, consulté le 23/04/2016 <http://www.colorado.edu/ studentgroups/libertarians/issues/friedman-soc-respbusiness.html>.

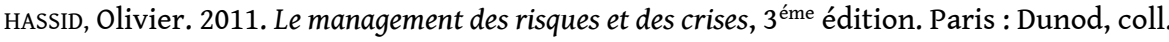
«Stratégie de l'Entreprise ».

HUMBLEY, John. 2006. « La néologie : interface entre ancien et nouveau ». In Greenstein Rosalind (dir.), Langues et cultures : une histoire d'interface? Paris : Publications de la Sorbonne, 91-103.

KLEINMAN, Daniel Lee \& Jack KLOPPENBURG. 1991. « Aiming for the discursive high ground: Monsanto and the biotechnology controversy ». Sociological Forum 6/3, 427-447.

MOIRAND, Sophie. 2004. «De la médiation à la médiatisation des faits scientifiques et techniques : où en est l'analyse du discours? ». Colloque Sciences, Médias et Société 15-17 juin 2004, Lyon, ENSLSH, <http://sciences-medias.ens-lyon.fr/article.php3?id_article=59>, consulté le 13 novembre 2016.

NALEBUFF, Adam \& Barry BRANDENBERGER. 1996. Co-opetition. New York : Currency/Doubleday.

OWEN, John R. \& Deanna KEMP. 2013. « Social licence and mining: A critical perspective ». Resources Policy, 38/1, 29-35.

Peynaud, Caroline. 2014. « Définition et explication dans la presse généraliste aux États-Unis : de la médiation du discours expert à la création d'un intertexte médiatique », ASp 65, 21-43.

PETIT, Michel. 2010. « Le discours spécialisé et le spécialisé du discours : repères pour l'analyse du discours en anglais de spécialité », E-rea 8/1, <http://erea.revues.org/1400>, consulté le $13 / 09 / 2016$

VALOR, Carmen. 2005. « Corporate social responsibility and corporate citizenship: Towards corporate accountability ». Business and Society Review 110/2, 191-212.

VAN DER YEUGHT, Michel. 2016. «A proposal to establish epistemological foundations for the study of specialised languages ». ASp 69, 41-63.

YAMI, Saïd (dir.). 2010. Coopetition: Winning Strategies for the 21st century. Northampton, MA :

Edward Elgar. 


\section{NOTES}

1. Sauf indication contraire, l'ensemble des pages d'accueil mentionnées dans le présent article a été consulté le 20 novembre 2015.

2. "I propose that specialised domains are sets of knowledge and practices which transcend their originators and are harnessed to the service of one particular purpose » (Van der Yeught $2016: 51$ ).

3. «Activities are largely subject to time and space determinations and remain attached to persons while purposes transcend local situations and are better suited to the establishment of objective knowledge " (Van der Yeught $2016: 51$ ).

4. < http://neinuclearnotes.blogspot.fr/2015/08/nuclear-energy-for-this-generation-and.html>, consulté le 9 septembre 2016.

5. <http://www.nei.org/>, consulté le 15 novembre 2015.

6. < http://neinuclearnotes.blogspot.fr/2015/08/nuclear-energy-for-this-generation-and.html>, consulté le 9 septembre 2016.

7. <https://www.chevron.com/stories/chevron-fellows>, consulté le 3 septembre 2016.

8. Ibid.

9. <http://www.merck.com/about/featured-stories/Tim_Cernak.html>, consulté le 17 novembre 2015.

10. < http://www.pfizer.com/research/science_and_technology/meet_our_scientists/ mikael_dolsten>, consulté le 30 novembre 2016.

11. < http://corporate.exxonmobil.com.qa/en-qa/environment/environmental-performance/ environmental-stewardship/overview>, consulté le 17 novembre 2015.

12. <http://www.monsanto.com/products/pages/breeding.aspx>, consulté le 17 novembre 2015.

13. <https:// www.chevron.com/-/media/chevron/shared/documents/EnergyAndOranges.pdf>, consulté le 17 novembre 2015.

14. Ibid.

15. <http://www.monsanto.com/products/pages/breeding.aspx>, consulté le 17 novembre 2015.

16. Corps gras ajouté par l'auteur.

17. Ibid.

18. <http://www.pfizer.com/news/video-gallery>, consulté le 17 novembre 2015.

19. <https://www.chevron.com/weagree/australia/>, consulté le 17 novembre 2015.

20. <https:// www.chevron.com/-/media/chevron/shared/documents/ShaleGasTranscript.pdf>, consulté le 17 novembre 2015.

21. Ibid.

22. <https://nuclear.duke-energy.com/about>, consulté le 03 septembre 2015.

23. <https://gmoanswers.com/spécialistes>, consulté le 17 novembre 2015.

24. <https://gmoanswers.com/ask/glyphosate-bonds-lipids-create-highly-reactivephospholipid-analog-lecithin-crosses-both-blood>, consulté le 17 novembre 2015.

25. C'est à Ray Noorda, le fondateur de Novell, que nous devons ce néologisme. Le terme fut ensuite popularisé par Adam Nalebuff et Barry Brandenburger.

\section{RÉSUMÉS}

Cette étude porte sur la visibilité croissante des chercheurs, des médecins ou des ingénieurs dans les actions de communication d'entreprises américaines spécialisées dans les secteurs de 
l'agrochimie, du pétrole, du nucléaire et de la pharmacie. Afin de caractériser la fonction de ces « spécialistes d'entreprise », deux corpus ont été analysés. Le premier corpus comprend les pages d'accueil des entreprises étudiées, mises en ligne entre 2000 et 2015. Le second corpus vise à caractériser des genres émergents récemment mis en place par les multinationales pour permettre aux spécialistes de présenter leur mission au sein de l'entreprise. Une analyse du contenu visuel et textuel a été effectuée pour chaque corpus. Ces analyses soulignent le rôle fondamental des spécialistes dans le processus de médiation des connaissances liées aux nouvelles technologies et la légitimation de techniques parfois controversées. En tant que membres de l'entreprise, les spécialistes contribuent également à la redéfinition de l'image de l'entreprise et de ses activités, à travers la promotion d'une science responsable, qui vise à améliorer les conditions de vie des plus vulnérables.

This study focuses on the growing importance of researchers, doctors or engineers in the online corporate discourse of American companies specialized in agrochemistry, oil and gas production, nuclear energy and pharmaceuticals. In order to characterize the function of corporate specialists, two corpora were analyzed. The first corpus comprises the homepages of the corporate websites available online between 2000 and 2015. The second corpus aims to characterize emerging web genres (interviews, video, product pages or blogs) where the specialists describe their mission within the company. For each corpus, analyses of the visual and textual content were carried out. Results highlight the essential role of specialists in circulating knowledge related to new technologies and to legitimize sometimes controversial techniques. As company members, specialists also contribute to redefining the company's identity and activities: they promote a responsible approach to science, seeking to improve the living conditions of the most vulnerable people.

\section{INDEX}

Keywords : online corporate discourse, specialized domain, legitimization, mediation, technological risk, specialist

Mots-clés : discours d'entreprise en ligne, domaine spécialisé, légitimation, médiation, risque technologique, spécialiste

\section{AUTEUR}

\section{FANNY DOMENEC}

Fanny Domenec est maître de conférences à l'Université Paris 2 où elle enseigne l'anglais économique et financier. Dans le cadre de l'analyse des domaines spécialisés, son travail de recherche, dans le cadre du CeLiSo (EA 7332), porte sur la spécificité du domaine du risque (technologique et financier) au sein de différents milieux spécialisés. Elle étudie notamment l'évolution des genres traditionnels du discours de l'entreprise et l'apparition de genres émergents en contexte de controverse. Elle s'intéresse également à la perception du risque par le grand public et à la médiation du discours sur le risque par différentes communautés professionnelles. fanny.domenec@u-paris2.fr 